\title{
Mammalian Target of Rapamycin Complex 2 Signaling Is Required for Liver Regeneration in a Cholestatic Liver Injury Murine Model
}

Yi Zhou, ${ }^{* \dagger}$ Meng Xu, ${ }^{\dagger \ddagger}$ Pin Liu ${ }^{\ddagger \S}$ Binyong Liang, ${ }^{\ddagger \top}$ Manning Qian, ${ }^{\ddagger \|}$ Haichuan Wang, ${ }^{\ddagger * * \dagger \dagger}$ Xinhua Song, Pranavanand Nyshadham, ${ }^{\ddagger}$ Li Che, ${ }^{\ddagger}$ Diego F. Calvisi, ${ }^{\S}$ Feng Li, ${ }^{\ddagger}$ Shumei Lin, ${ }^{*}$ and Xin Chen

\begin{abstract}
From the Department of Infectious Diseases, * The First Affiliated Hospital of Xi'an Jiaotong University, Xi'an, PR China; the Department of Bioengineering and Therapeutic Sciences and Liver Center, ${ }^{\dagger}$ University of California, San Francisco, California; the Department of General Surgery, ${ }^{\ddagger}$ The Second Affiliated Hospital of Xi'an Jiaotong University, Xi'an, PR China; the Department of Pediatrics, ${ }^{\S}$ Zhongnan Hospital of Wuhan University, Wuhan, PR China; the Hepatic Surgery Center, ${ }^{\top}$ Department of Surgery, Tongji Hospital, Tongji Medical College, Huazhong University of Science and Technology, Wuhan, PR China; the Clinical Medical College of Yangzhou University," Yangzhou, PR China; the Liver Transplantation Division, ** Department of Liver Surgery, and the Laboratory of Liver Surgery, ${ }^{\dagger \dagger}$ West China Hospital, Sichuan University, Chengdu, PR China; the Center for Drug Discovery, ${ }^{\ddagger \ddagger}$ Department of Pathology and Immunology, Baylor College of Medicine, Houston, Texas; and the Institute of Pathology, ${ }^{\S \S}$ University of Regensburg, Regensburg, Germany
\end{abstract}

Accepted for publication

March 25, 2020.

Address correspondence to

Shumei Lin, M.D., Ph.D.,

Department of Infectious Diseases, The First Affiliated Hospital of Xi'an Jiaotong

University, No. 277 Yanta West Road, Xi'an, Shaanxi, PR China, 710061; or Xin Chen, Ph.D., Department of Bioengineering and Therapeutic Sciences, University of California, San Francisco, 513 Parnassus Ave., S-816, San Francisco, CA 94143. E-mail: 1smxjtu@126. com orxin.chen@ucsf.edu.
Cholestatic liver injury may lead to a series of hepatobiliary syndromes, which can progress to cirrhosis and impaired liver regeneration, eventually resulting in liver-related death. Mammalian target of rapamycin complex 2 (mTORC2) is a major regulator of liver metabolism and tumor development. However, the role of mTORC2 signaling in cholestatic liver injury has not been characterized to date. In this study, we generated liver-specific Rictor knockout mice to block the mTORC2 signaling pathway. Mice were treated with 3,5-diethoxycarbonyl-1,4-dihydrocollidine (DDC) to induce cholestatic liver injury. DDC feeding induced cholestatic liver injury and ductular reaction as well as activation of the mTORC2/Akt signaling pathway in wild-type mice. Loss of mTORC2 led to significantly decreased oval cell expansion after DDC feeding. Mechanistically, this phenotype was independent of mTORC1/fatty acid synthase cascade (Fasn) or yes-associated protein (Yap) signaling. Notch pathway was instead strongly inhibited during DDC-induced cholestatic liver injury in liver-specific Rictor knockout mice. Furthermore, mTORC2 deficiency in adult hepatocytes did not inhibit ductular reaction in this cholestatic live injury mouse model. Our results indicated that mTORC2 signaling effectively regulates liver regeneration by inducing oval cell proliferation. Liver progenitor cells or bile duct cells, rather than mature hepatocytes, would be the major source of ductular reaction in DDC-induced cholestatic liver injury. (Am J Pathol 2020, 190: 1414-1426; https://doi.org/10.1016/j.ajpath.2020.03.010)
Cholestatic liver disease is a serious hepatic illness resulting from impaired bile secretion by hepatocytes or intrahepatic/ extrahepatic bile duct obstruction. In this disease, although the liver has a good capacity to repair, injuries to bile ducts or hepatocytes may also lead to a wide spectrum of adverse consequences, ranging from hepatic inflammation to fibrogenesis with progression to cirrhosis and hepatobiliary cancer. $^{1,2}$

The pathogenesis of cholestatic liver injury is complex and not fully elucidated. In the past 25 years, multiple animal models have been established to understand the mechanisms and seek new treatment strategies for this disease. ${ }^{3}$ Among them, the 3,5-diethoxycarbonyl-1,4-dihydrocollidine (DDC)feeding mouse is a well-established model of cholestatic liver injury. Specifically, DDC feeding leads to increased biliary

\footnotetext{
Y.Z. and M.X. contributed equally to this work.

Supported by NIH grants R01CA239251 (X.C.), R01DK121970 (F.L.), and P30DK026743 (University of California, San Francisco, Liver Center); National Natural Science Foundation of China grant 81902449 (M.X.); and China Scholarship Council scholarship contract number 201806280217 (Y.Z.).
}

Disclosures: None declared. 
porphyrin secretion and subsequently induces sclerosing cholangitis. A previous study showed that DDC feeding increases profibrotic and proinflammatory cytokine expression and activates periductal myofibroblasts around bile ducts. ${ }^{4}$ In addition, Fickert et $\mathrm{al}^{5}$ reported that DDC treatment in mice leads to the generation of intraductal pigment plugs and intense pericholangitis, as well as segmental bile duct obstruction. More important, the DDC-induced cholangiopathy is accompanied by ductular reaction and oval cell expansion. Thus, chronic DDC feeding in mice has been used as an ideal model for the study of oval cell-mediated liver regeneration. ${ }^{6}$ However, the molecular mechanisms of biliary repair and how cholestatic disease progresses are still poorly understood.

It is well known that the mammalian target of rapamycin (mTOR) signaling pathway plays a significant role in liver injury and regeneration. ${ }^{7,8}$ mTOR belongs to the phosphatidylinositol 3-kinase-related kinase family of protein kinases and serves as a core component of two distinct protein complexes, mTORC1 and mTORC2, which phosphorylate a different set of substrates to regulate many fundamental cell processes. mTORC1 is composed of three core components (mTOR, Raptor, and mIST8), and it plays a central role in regulating protein synthesis, nucleotide biosynthesis, lipogenesis, glycolysis, and autophagy. There are two major cascades downstream of mTORC1: eukaryotic translation initiation factor 4E-binding protein 1 (4EBP1)/eukaryotic translation initiation factor 4E cascade, which regulates protein synthesis; and p70S6K/RPS6 cascade, which in turn regulates SREBP1 and fatty acid synthase (FASN) and the related de novo lipogenesis. ${ }^{9}$ Instead of Raptor, rapamycin-insensitive companion of mTOR (Rictor) is the core protein of mTORC2, and ablation of Rictor has been demonstrated to block mTORC2 signaling pathway. ${ }^{10,11}$ Through phosphorylating and activating several AGC subfamily kinases, including AKT, serum and glucocorticoid induced kinase (SGK), and protein kinase $\mathrm{C}, \mathrm{mTORC} 2$ controls the survival and growth by regulating cell proliferation, metabolism, and the activities of the cytoskeleton. ${ }^{12}$ Among all these molecules, AKT kinases have been considered the major substrate of mTORC2. ${ }^{13}$ In a previous study, it was shown that phosphorylated (p)-AKT could be detected in samples of human primary biliary cirrhosis, ${ }^{14}$ a major type of chronic cholestatic liver disease. The results suggest the activation of mTORC2 in this pathologic condition in humans. However, the role of mTORC2 signaling in development of cholestatic liver injury and liver regeneration has not been investigated to date.

In this article, we report the generation of a mouse model with liver-specific mTORC2 knockout obtained by deletion of Rictor in the mouse liver. In these mice, we evaluated DDC-induced liver injury and oval cell expansion. The results demonstrate that loss of mTORC2 inhibits hepatic progenitor cell- or bile duct cell-mediated, but not mature hepatocyte-dependent, oval cell expansion by regulating the Akt function. Mechanistically, we found that
mTORC2-modulated oval cell expansion is independent of the Rps6/Fasn cascade, while presumably being mediated by the Notch signaling pathway. Altogether, our data provide novel insights into the role of mTORC2 in regulating liver regeneration during chronic liver injury.

\section{Materials and Methods}

\section{Reagents}

Adeno-associated virus encoding Cre-recombinase under the control of hepatocyte-specific thyroxine-binding globulin promoter (AAV-TBG-Cre) and AAV-TBG-Null virus were obtained from the University of Pennsylvania Gene Therapy Core Services (Philadelphia, PA). The 0.1\% DDCsupplemented diet was produced by Research Diets (New Brunswick, NJ). Rapamycin (LC Laboratories, Woburn, MA) was dissolved in $100 \%$ ethanol to make $50 \mathrm{mg} / \mathrm{mL}$ rapamycin stock, then mixed with 10\% PEG400 and $10 \%$ Tween 80 to make $1 \mathrm{mg} / \mathrm{mL}$ working solution.

\section{Animals and Treatments}

FVB/N mice, Albumin-Cre transgenic mice, ${ }^{15}$ and Rictor ${ }^{A / A l}$ mice $^{16}$ (all in the C57BL/6 background) were obtained from Jackson Laboratory (Bar Harbor, ME). Fasn ${ }^{f / f l}$ mice $^{17}$ (in the C57BL/6 background) were kindly provided by Dr. Clay F. Semenkovich (Washington University, St. Louis, MO). Rictor ${ }^{f l / f}$ mice and $F a s n^{f l / l}$ mice were crossed with Albumin-Cre to generate liver-specific Rictor knockout mice (Rictor ${ }^{L K O}$ ) and liver-specific Fasn knockout mice $\left(F_{a s n}{ }^{L K O}\right.$ mice), respectively. Genotyping was conducted using the Mouse Direct PCR Kit (catalog number B40015; Bimake, Houston, TX), according to the standard protocol. To deplete the Rictor gene specifically in adult mouse hepatocytes, Rictor ${ }^{f / f l}$ mice (aged 6 weeks) were infected with $4 \times 10^{11}$ genome copies AAV-TBG-Cre vectors by tail vein injection and aged for 3 weeks to allow the complete deletion of the gene in adult hepatocytes. An AAV-TBGNull vector was used as the control. Both male and female mice were used in the study.

DDC treatment in mice was performed as described in previous studies. ${ }^{18}$ In brief, 6- to 9-week-old mice were fed a $0.1 \%$ DDC-supplemented diet for 3 weeks. Standard diet and age- and sex-matched mice were used as controls. An additional group of mice was fed a $0.1 \%$ DDCsupplemented diet for 3 weeks, followed by 3 weeks of recovery with standard chow diet. To block the mTORC1 pathway, rapamycin was intraperitoneally injected in a dose of $6 \mathrm{mg} / \mathrm{kg}$ per day for 6 days a week and subjected to DDC diet for 3 weeks in wild-type mice. Mice were injected intraperitoneally with the vehicle (PEG400 and Tween 80) as controls. After 3 weeks of feeding, mice were euthanized. Liver weight was recorded, and the liver/body weight ratio was calculated. Blood samples and liver tissues were collected. Animal experiments were performed according to 
protocols approved by the Committee for Animal Research at the University of California, San Francisco (San Francisco, CA).

\section{Biochemical Assays}

The serum alanine/aspartate aminotransferase, alkaline phosphatase, total bilirubin/direct bilirubin, and albumin levels were assayed by the Clinical Laboratory of the Zuckerberg San Francisco General Hospital (San Francisco, CA).

\section{Histology and Immunohistochemistry}

Liver samples were fixed overnight in 10\% neutral-buffered formalin (Thermo Fisher Scientific, Waltham, MA), embedded in paraffin, cut into sections ( $5 \mu \mathrm{m}$ thick), and collected on glass slides. The sections were stained with hematoxylin and eosin, using standard procedures. For immunohistochemistry, liver sections were processed for antigen retrieval in $10 \mathrm{mmol} / \mathrm{L}$ sodium citrate buffer (microwaved, 10 minutes) after deparaffinization and rehydration. Subsequently, slides were permeabilized with $1 \times$ phosphate-buffered saline, then blocked with $5 \%$ goat serum, followed by incubation with primary antibodies overnight at $4^{\circ} \mathrm{C}$. The anti-cytokeratin 19 (CK19; 52625; Abcam, Cambridge, UK), anti-sex determining region Y box 9 (Sox9; 82630; Cell Signaling Technology, Danvers, MA), anti- $\alpha$-smooth muscle actin ( $\alpha$-SMA; M0851; Agilent, Santa Clara, CA), anti-vimentin (5741; Cell Signaling Technology), anti-Ki-67 (MA5-14520; Thermo Fisher Scientific), anti-F4/80 (14-4801-82; eBioscience, San Diego, CA), and anti-YAP (4912; Cell Signaling Technology) antibodies were used. Endogenous peroxidase activity was blocked by 20 minutes of incubation of the slides in 3\% $\mathrm{H}_{2} \mathrm{O}_{2}$. Subsequently, slides were incubated with the biotinconjugated secondary antibody (B2770; Thermo Fisher Scientific) for 1 hour at room temperature. Finally, the immunoreactivity was visualized using the Vectastain Elite $\mathrm{ABC}$ Kit (Vector Laboratories, Burlingame, CA) and diaminobenzidine substrates (Dako North America, Carpinteria, CA). Slides were counterstained with Mayer's hematoxylin. Images were taken using a bright-field microscope connected to a digital color camera (Leica, San Francisco, CA; model DFC295). The quantification was performed using the ImageJ version 1.46r $(\mathrm{NIH}$, Bethesda, MD; http://imagej.nih.gov/ij) and Image Pro Plus software version 6.0 (Media Cybernetics, Rockville, MD).

\section{Picro Sirius Red Staining}

Liver tissues were embedded in paraffin, cut into sections (5 $\mu \mathrm{m}$ thick), and collected on glass slides, which were placed in an oven at $60^{\circ} \mathrm{C}$ for 45 minutes. Picro Sirius Red stain solution was prepared as follows: $0.1 \%$ direct red 80 plus $0.1 \%$ fast green FCF dissolved in $1.2 \%$ picric acid. After deparaffinization and rehydration, slides were incubated with Picro Sirius Red solution for 60 minutes. Next, slides were quickly dehydrated and mounted with clean mounting medium.

\section{Immunofluorescence Staining}

Frozen liver tissues were embedded in Tissue-Tek OCT compound (Sakura, Staufen, Germany), cut into sections (5 $\mu \mathrm{m}$ thick), and fixed in $4 \%$ paraformaldehyde (Sigma, St. Louis, MO) for 10 minutes at room temperature. After washing with phosphate-buffered saline, the sections were blocked with $5 \%$ goat serum and $0.1 \%$ Triton X-100 (in phosphate-buffered saline) for 30 minutes. Next, the sections were incubated with primary antibodies [A6, epithelial cell adhesion molecule (EpCAM), and CK19] overnight at $4^{\circ} \mathrm{C}$. The A6 antibody was kindly supplied by Dr. Valentina M. Factor (Laboratory of Experimental Carcinogenesis, National Cancer Institute, NIH, Bethesda, MD). The EpCAM antibody was purchased from Biolegend (118201; Biolegend, San Diego, CA), and the CK19 antibody was purchased from Abcam (52625). The following day, after washing with phosphate-buffered saline, sections were incubated with Alexa Fluor 488 and/or Alexa Fluor 594 secondary antibodies for 30 minutes in the dark. Finally, the sections were mounted with VECTASHIELDH Mounting Medium with DAPI (Vector Laboratories), and the immunofluorescence signal was visualized by an immunofluorescence microscope.

\section{Protein Extraction and Western Blot Analysis}

Frozen mouse liver specimens were homogenized in Mammalian Protein Extraction Reagent (Thermo Fisher Scientific) containing the Complete Protease Inhibitor Cocktail (Thermo Fisher Scientific). The lysate was clarified by centrifugation $16,000 \times g$ for 10 minutes at $4^{\circ} \mathrm{C}$ ) and stored at $-80^{\circ} \mathrm{C}$ until assayed. Protein concentrations were determined using the Bio-Rad Protein Assay Kit (Bio-Rad, Hercules, CA), and bovine serum albumin was used as standard. Aliquots of $30 \mu \mathrm{g}$ protein lysates were denatured by boiling in Tris-Glycine SDS Sample Buffer (Invitrogen, Grand Island, NY), separated by SDS-PAGE, transferred onto nitrocellulose membranes (Invitrogen), blocked in 5\% nonfat dry milk, and incubated overnight at $4{ }^{\circ} \mathrm{C}$ with the specific primary antibodies. Each primary antibody was followed by incubation with a horseradish peroxidase secondary antibody diluted 1:5000 for 1 hour and revealed with the Super Signal West Pico Chemiluminescent Substrate (Pierce Chemical Co, New York, NY). The following antibodies were used: Rictor (9476), phosphorylated (phospho)-AKT (Ser473; 3787), phospho-AKT (Ser308; 13038), total AKT (9272), phospho-Akt1 (Ser473; 9018), phospho-Akt2 (Ser474; 8599), phosphoforkhead box O1 (p-FoxO1; Ser256; 84192), phospho-proline-rich AKT substrate of $40 \mathrm{kDa}$ (p-PRAS40; Thr246; 2997), phospho-mTOR (Ser2448; 2971), phospho-S6 
ribosomal protein (Ser235/236; 4858), phospho-4EBP1 (Ser65; 9451), YAP/transcriptional coactivator with PDZbinding motif (TAZ) (8418), phospho- $\beta$-catenin (Ser552; 5651), FASN (3180), and glyceraldehyde-3-phosphate dehydrogenase (5174) from Cell Signaling Technology. The SOX9 (ab185230), cytokeratin 19 (ab52625), and Jagged1 (ab109536) antibodies were purchased from Abcam. The Sin1 antibody (A300-910A) was purchased from Bethyl Laboratories (Montgomery, TX).

\section{RNA Extraction and RT-qPCR}

Total mRNA was extracted from liver and small intestine tissues using the Quick RNA miniprep kit (Zymo Research, Irvine, CA). mRNA expression was determined by quantitative RT-PCR (RT-qPCR) using the SYBR Green Master Mix (Applied Biosystems, Foster City, CA) in a QuantStudio 6 Flex system (Applied Biosystems). Expression of each gene was normalized to the $18 \mathrm{~S}$ rRNA. The primer sequences are listed in Table 1.

\section{Bile Acid Analysis}

Total bile acid levels in serum and liver lysate samples were measured using the Mouse Total Bile Acids Assay Kit (80470; Crystal Chem, Elk Grove Village, IL), following the manufacturer's protocol. The analysis was performed with the BioTek ELx808 Absorbance Reader (BioTek, Winooski, VT), and the results were shown as $\mu \mathrm{mol} / \mathrm{L}$. Ultra-high-performance liquid chromatography-tandem mass spectrometry analysis was conducted to profile the taurocholic acid and deoxycholic acid levels in samples obtained from DDC fed mice. Serum $(20 \mu \mathrm{L})$ was added to $60 \mu \mathrm{L}$ of methanol, followed by vortexing and centrifugation at $15,000 \times g$ for 15 minutes. Livers were weighed and homogenized in three times volume of aqueous methanol. Subsequently, $50 \mu \mathrm{L}$ of each mixture was added to $200 \mu \mathrm{L}$ of methanol, followed by vortexing and centrifugation at $15,000 \times g$ for 15 minutes. Each supernatant was transferred to a sample vial for analysis. Supernatant ( $3 \mu \mathrm{L}$ ) was injected into an ultra-highperformance liquid chromatography-tandem mass spectrometry instrument coupled with Q Exactive Orbitrap MS (Thermo Fisher Scientific) equipped with a $2.1 \times 100-\mathrm{mm}$

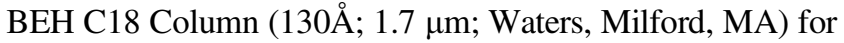
analysis. The column temperature was maintained at $40^{\circ} \mathrm{C}$. The flow rate of mobile phase was $0.3 \mathrm{~mL} /$ minute, with a gradient ranging from $2 \%$ to $98 \%$ aqueous acetonitrile containing $0.1 \%$ formic acid in a 7-minute run. Q Exactive MS was operated in negative mode with electrospray ionization. Ultrapure nitrogen was applied as the sheath (45 arbitrary units), auxiliary (10 arbitrary units), sweep (1.0 arbitrary units), and the collision gas. The capillary gas temperature was set at $275^{\circ} \mathrm{C}$, and the capillary voltage was set at $3.7 \mathrm{kV}$. Mass spectrometry data were acquired from $\mathrm{m} / \mathrm{z} 80$ to $1200 \mathrm{Da}$ in profile mode, and reference ions at $\mathrm{m} / \mathrm{z} 174.9562$ in the negative mode were used as a lock mass during acquisition.

\section{Statistical Analysis}

The Prism software version 6.0 (GraphPad, San Diego, CA) was applied to analyze the data, which are presented as means \pm SEM. Comparisons between two groups were performed with the two-tailed unpaired $t$-test. $P<0.05$ was considered statistically significant.

\section{Results}

DDC Ingestion Leads to Cholestatic Liver Injury and Induces mTORC2/Akt Activation

As the first step to investigate mTORC2/Akt signaling in cholestatic liver injury, wild-type mice were fed a regular chow diet or a $0.1 \%$ DDC-supplemented diet (DDC diet) for 3 weeks (Figure 1A). Consistent with a previous report, ${ }^{19}$

Table 1 Primer Information for RT-qPCR

\begin{tabular}{|c|c|c|}
\hline Gene & Forward & Reverse \\
\hline 18S rRNA & $5^{\prime}$-CGGCTACCACATCCAAGGAA-3' & $5^{\prime}$-GCTGGAATTACCGCGGCT-3' \\
\hline Mouse: Hes1 & 5'-AAAGCCTATCATGGAGAAGAGGCG-3 & $5^{\prime}$-GGAATGCCGGGAGCTATCTTTCTT-3' \\
\hline Mouse: HeyL & $5^{\prime}$-CAGCCCTTCGCAGATGCAA-3' & $5^{\prime}$-CCAATCGTCGCAATTCAGAAAG- $3^{\prime}$ \\
\hline Mouse: Jagged1 & 5'-CCTCGGGTCAGTTTGAGCTG-3' & $5^{\prime}$-CCTTGAGGCACACTTTGAAGTA-3' \\
\hline Mouse: Fgf15 & $5^{\prime}-$ ATGGCGAGAAAGTGGAACGG-3' & $5^{\prime}$-CTGACACAGACTGGGATTGCT-3' \\
\hline Mouse: $P c$ & $5^{\prime}$-CTGAAGTTCCAAACAGTTCGAGG- $3^{\prime}$ & $5^{\prime}-\mathrm{CGCACGAAACACTCGGATG-3^{ \prime }}$ \\
\hline Mouse: Pck1 & 5'-CTGCATAACGGTCTGGACTTC-3' & $5^{\prime}$-CAGCAACTGCCCGTACTCC-3' \\
\hline Mouse: G6pc & 5'-CGACTCGCTATCTCCAAGTGA-3' & $5^{\prime}$-GTTGAACCAGTCTCCGACCA-3' \\
\hline Mouse: Srebf1 & 5'-CTGGTGAGTGGAGGGACCAT-3' & 5'-GACCGGTAGCGCTTCTCAAT-3' \\
\hline Mouse: Acly & $5^{\prime}$-CAGCCAAGGCAATTTCAGAGC-3' & $5^{\prime}$-CTCGACGTTTGATTAACTGGTCT-3' \\
\hline
\end{tabular}

RT-qPCR, quantitative RT-PCR. 
A
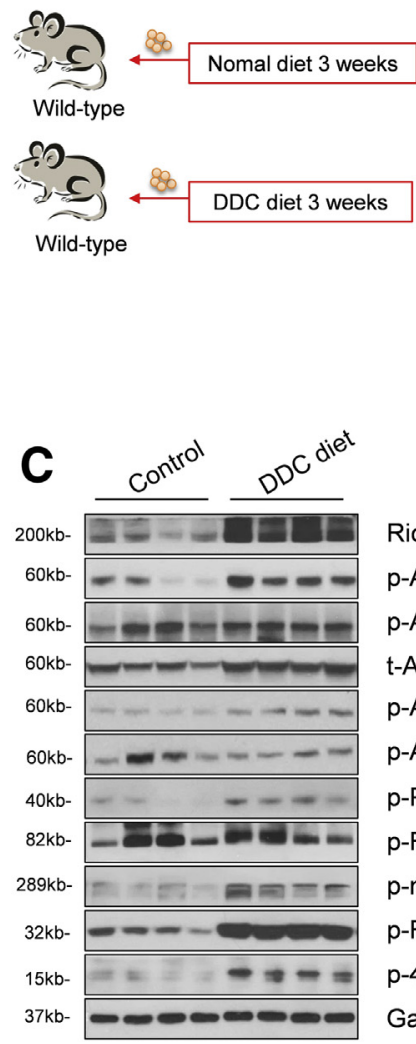

Rictor

p-Akt ${ }^{5473}$

$p-A k t^{\top 308}$

t-Akt

p-Akt1

p-Akt2

p-Pras 40

p-FoxO1

p-mTOR ${ }^{52448}$

p-Rps6s235/236

p-4Ebp1 ${ }^{\text {s65 }}$

Gapdh

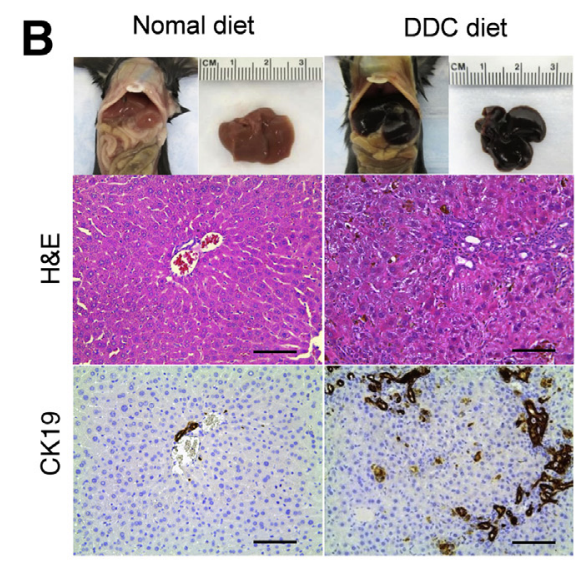

D

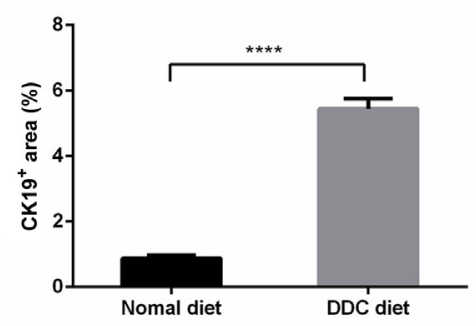

Figure 1 3,5-diethoxycarbonyl-1,4-dihydrocollidine (DDC) feeding leads to cholestatic liver injury and is accompanied by Akt activation. A: Scheme of the experiment. B: Gross image and hematoxylin and eosin (H\&E) and immunohistochemical staining of cytokeratin 19 (CK19) proteins in the livers of Rictor ${ }^{f l f l}$ mice with or without DDC diet. C: Western blot analysis of the mTORC2/ Akt signaling cascade in livers of Rictor ${ }^{f l f l}$ mice with or without DDC diet. Glyceraldehyde-3phosphate dehydrogenase (Gapdh) was used as loading control. At least three mice per group were assayed. D: Quantification of CK19 staining. At least three mice per group were assayed. Data are expressed as means \pm SEM (D). ${ }^{* * * * P}<0.0001$. Scale bars $=100 \mu \mathrm{m}$ (B). 4Ebp1, eukaryotic translation initiation factor 4E-binding protein; Fox01, forkhead box 01; mTOR, mammalian target of rapamycin; p-, phosphorylated; Pras40, prolinerich AKT substrate of $40 \mathrm{kDa}$; Rictor, rapamycininsensitive companion of mTOR; Rps6, ribosomal protein $\mathrm{S6}$; t-, total. grossly, liver from DDC fed mice appeared to be darker (Figure 1B). Histologic examination revealed that DDC feeding induced pigment plug formation in bile ducts, intrahepatic bile duct dilation, and inflammatory cell infiltration (Figure 1B). As CK19 is a widely used marker of biliary epithelial cells and oval cells ${ }^{20}$ CK19 expression was determined in control and DDC-fed livers by immunohistochemistry. A significantly increased number of CK19-positive cells were found in DDC-fed mouse liver tissues (Figure 1, B and D). Next, the activation of the mTORC2/Akt signaling in control and DDC-fed mouse liver tissues was investigated. DDC treatment led to an increased expression of Rictor and $\mathrm{p}-\mathrm{Akt}^{\mathrm{S} 473}$, supporting the activation of mTORC2. Interestingly, the p-Akt1 isoform, but not p-Akt2, was found to be activated in this cholestatic liver injury model. Furthermore, the downstream effectors of the mTORC2/Akt cascade, including $p$ Pras $40^{\mathrm{T} 246}, \mathrm{p}^{-\mathrm{mTOR}}{ }^{\mathrm{S} 2448}, \mathrm{p}-\mathrm{Rps}^{\mathrm{S} 235 / 236}$, and $\mathrm{p}-4 \mathrm{Ebp}^{\mathrm{S} 65}{ }^{\text {, }}$ but not p-FoxO1 ${ }^{\mathrm{S} 256}$, were expressed at higher levels in DDC-treated liver tissues (Figure 1C and Supplemental Figure S1A). The results indicated that DDC feeding induces mice cholestatic liver injury and ductular reaction. These events are paralleled by the activation of mTORC2/ Akt/mTORC1 signaling cascade.

\section{Genetic Depletion of Rictor Impairs Oval Cell Expansion in Mice}

To investigate the functional roles of mTORC2 in regulating cholestatic liver injury, liver-specific conditional Rictor gene knockout mice were generated. Specifically, the Albumin-Cre transgenic mouse that contained Cre recombinase with albumin promoter was crossed with the Rictor ${ }^{A / f l}$ mouse to restrict gene expression to liver hepatoblasts (progenitors of both biliary epithelial cells and hepatocytes) ${ }^{21}$ Genotyping was performed to validate the depletion of Rictor in mice by PCR analysis. Consistent with previous reports, ${ }^{21}$ the liver weight and liver weight/body weight ratio were slightly decreased in 6-week-old Rictor ${ }^{L K O}$ mice compared with Rictor $^{\text {flf }}$ littermates (Supplemental Figure S2, A and B). This phenotype is due to the decreased glycogen and triglyceride accumulation in the liver of Rictor ${ }^{L K O}$ mice. ${ }^{21}$ Histologic evaluation revealed that Rictor $^{L K O}$ mouse livers were completely normal. More important, we confirmed that bile duct development, as revealed by CK19 immunostaining, was similar in Rictor $^{L K O}$ and Rictor ${ }^{f / f t}$ control mice (Supplemental Figure S2C). The results suggest that mTORC2/Akt signaling is not required for normal liver development, including bile duct formation. 
Subsequently, Rictor $^{L K O}$ and Rictor ${ }^{A l / f}$ control littermates were fed a DDC-supplemented diet for 3 weeks (Figure 2A). Grossly, liver tissues appeared to be darker in all DDC-fed mice (Figure 2B). To ensure that loss of mTORC2 did not affect DDC-induced liver injury in mice, the liver biomarkers in the serum of the two cohorts of mice feeding regular chow or DDC were analyzed. DDC treatment led to significantly increased serum concentration of alanine aminotransferase, aspartate aminotransferase, total bilirubin, and direct bilirubin, without affecting total protein and albumin levels (Figure 3). More important, overall, similar results were obtained in both control and Rictor ${ }^{L K O}$ mice. In addition, taurocholic acid, deoxycholic acid, and total bile acid levels were similar in serum and liver tissues from Rictor $^{L K O}$ and Rictor $^{\text {flfl }}$ mice (Supplemental Figure S3); and gut fibroblast growth factor 15 (Fgf15) expression levels were also similar in two mouse cohorts (Supplemental Figure S4A). These data suggest that DDC treatment is effective in inducing liver injury in both strains of mice.

Next, histologic analysis of the liver tissues from DDCfed Rictor ${ }^{L K O}$ mice as well as Rictor ${ }^{f l f l}$ control littermates was performed. A similar pigment plug formation in the liver tissues of the two groups of mice was detected. As described previously, ${ }^{18,22}$ in DDC-treated Rictor ${ }^{f / f l}$ mice, a population of small cells with high nucleus/cytoplasm ratio as well as an atypical duct-like morphology was observed in the periportal area of the liver. In contrast, Rictor $^{L K O}$ mice showed few of these atypical duct-like cells at the periportal region of the liver (Figure 2B). To further investigate this phenotype, immunohistochemical staining of CK19 on liver tissues was conducted. Consistent with the histologic evaluation, CK19 $(+)$ cells were significantly decreased in DDC-treated Rictor ${ }^{L K O}$ mouse liver tissues (Figure 2, B and C). Additionally, immunohistochemistry of Sox9 (Figure 2B) as well as immunofluorescence of EpCAM and the oval cell marker A6 (Figure 4, A and B) were also performed. The expression of these markers was augmented in the atypical duct-like cells in DDC-treated Rictor $^{f / A}$ mice, whereas few cells expressed these oval cell markers in DDC-treated Rictor ${ }^{L K O}$ mice (Figures 2, B, D, and E, and 4, $\mathrm{A}$ and B). Double immunofluorescence staining of CK19 and A6 showed that the two markers were co-expressed in the same set of cells in Rictor ${ }^{\text {Alfl }}$ and Rictor ${ }^{L K O}$ mouse liver tissues (Figure 4C).

To elucidate the mechanisms leading to the reduced oval cell expansion in Rictor ${ }^{L K O}$ mice, we determined whether loss of mTORC2 affects DDC-induced liver fibrosis and hepatocyte proliferation. Thus, DDC-treated Rictor ${ }^{A / f l}$ and Rictor $^{L K O}$ mouse liver tissues were stained with Picro Sirius Red as well as anti- $\alpha$-smooth muscle actin and anti-
A

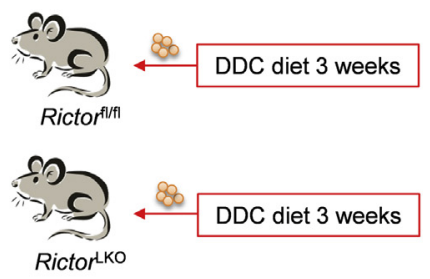

B

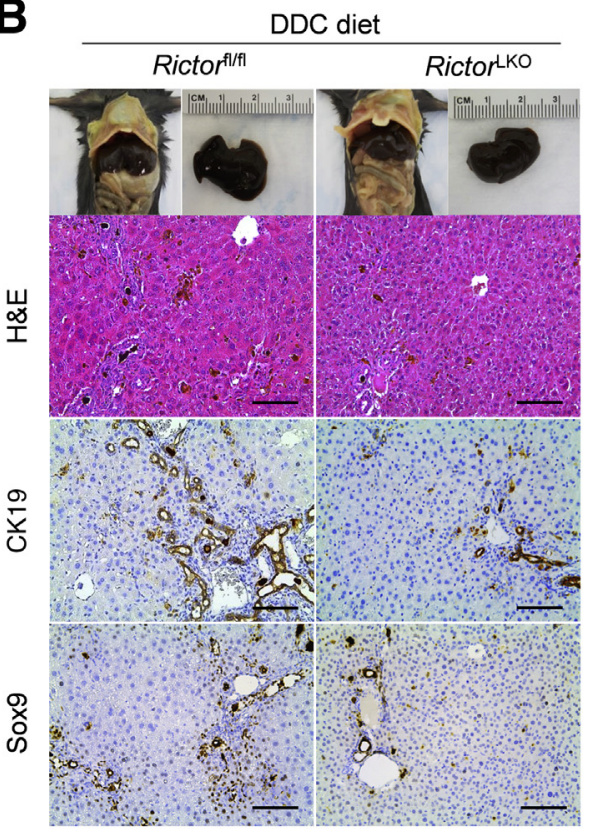

D
E

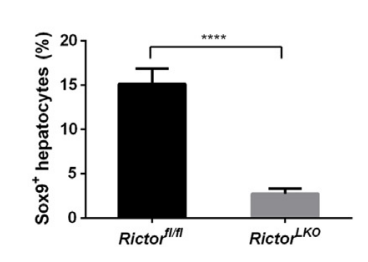

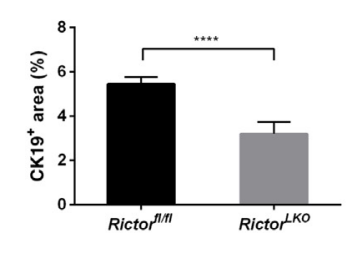

C

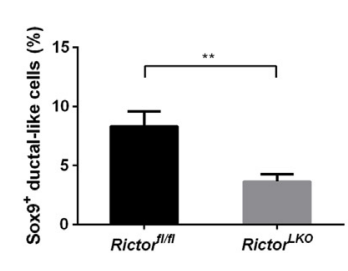

Figure 2 Genetic Rictor ablation impairs ductular reaction. A: Scheme of the experiment. B: Gross image and hematoxylin and eosin (H\&E) and immunohistochemical staining of cytokeratin 19 (CK19) and sex determining region Y-box 9 (Sox9) proteins in the livers of Rictor ${ }^{f / f l}$ and Rictor $^{\text {LKO }}$ mice fed 3,5-diethoxycarbonyl-1,4-dihydrocollidine (DDC) diet. C-E: Quantification of CK19 (C), Sox $9^{+}$ductular-like cell (D), and So $\times 9^{+}$ hepatocyte (E) staining. At least three mice per group were assayed. Data are expressed as means \pm SEM $(\mathbf{C}-\mathbf{E}) . \quad * * P<0.01$, $* * * * P<0.0001$. Scale bars $=100 \mu \mathrm{m}(\mathbf{B})$. 

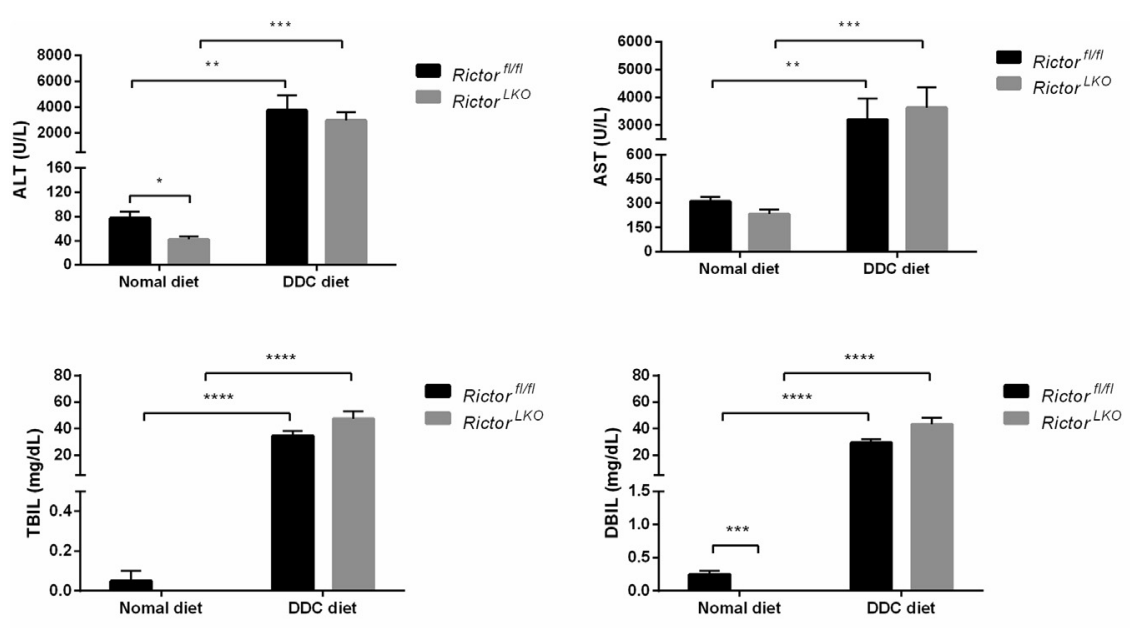

Figure 3 Indicators of liver function in Rictor $^{f l f l}$ and Rictor ${ }^{\perp K O}$ mice subjected or not to 3,5-diethoxycarbonyl-1,4-dihydrocollidine (DDC) feeding. At least three mice per group were assayed. Data are expressed as means \pm SEM. ${ }^{*} P<0.05,{ }^{* *} P<0.01,{ }^{* *} P<0.001$, and $* * * * P<0.0001$. ALB, albumin; ALT, alanine aminotransferase; AST, aspartate aminotransferase; DBIL, direct bilirubin; TBIL, total bilirubin; TP, total protein.
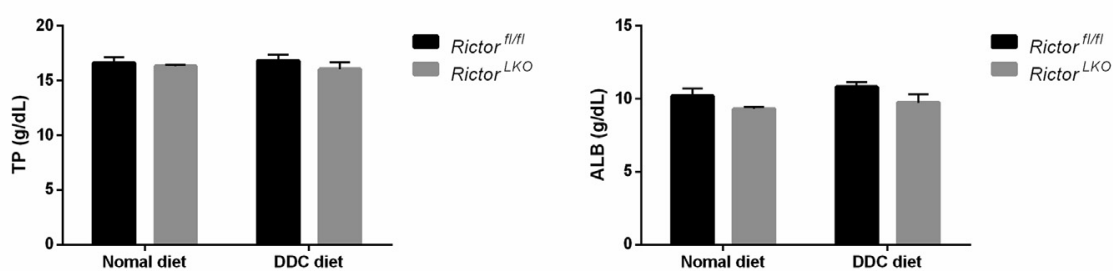

vimentin antibodies (Figure 5A). A significant expansion of Sirius Red $(+), \alpha$-smooth muscle actin $(+)$, and vimentin $(+)$ cells in DDC-treated Rictor ${ }^{f l f l}$ and Rictor $^{L K O}$ mouse livers was detected when compared with regular chow fed mice. Interestingly, Picro Sirius Red staining was slightly decreased and vimentin staining was significantly decreased in DDC-treated Rictor ${ }^{L K O}$ mouse liver tissues (Figure 5, B and $\mathrm{C}$ ), whereas $\alpha$-smooth muscle actin staining pattern was similar in Rictor $^{\text {flfl }}$ and Rictor ${ }^{L K O}$ mouse liver tissues (Figure 5D). In addition, there was a significant decrease of F4/80 (+) macrophages in DDC-treated Rictor ${ }^{L K O}$ mouse liver tissues (Supplemental Figure S5). Using immunohistochemical staining of $\mathrm{Ki}-67$, the hepatocyte proliferation rate in mouse livers was quantified. No difference was detected between Rictor $^{\text {fl/fl }}$ and Rictor ${ }^{L K O}$ mice (Figure 5E). These data suggest that loss of mTORC2 does not affect DDC treatment-induced hepatocyte proliferation, but it reduces liver fibrosis and inflammation after DDC-induced liver injury.

Next, Western blot analysis of liver tissues from Rictor ${ }^{\text {flf }}$ and Rictor ${ }^{L K O}$ mice on regular chow or DDC chow diet was performed. As expected, Rictor protein expression was completely lost in Rictor $^{L K O}$ mouse liver tissues (Figure 6 and Supplemental Figure S1B). This event was accompanied by a significantly lower expression of $\mathrm{p}-\mathrm{Akt}^{\mathrm{S} 473}$, but not $\mathrm{p}-\mathrm{Akt}^{\mathrm{T} 308}$, in DDC-treated Rictor $^{L K O}$ mouse livers (Figure 6 and Supplemental Figure S1B). Levels of mTORC1, the major downstream effector of Akt, were significantly decreased in Rictor ${ }^{L K O}$ livers, as demonstrated by the down-regulation of $\mathrm{p}-\mathrm{mTOR}^{\mathrm{S} 2448}, \mathrm{p}-4 \mathrm{Ebp}^{\mathrm{S} 65}{ }^{\text {, and }}$
p-Rps6 ${ }^{\mathrm{S} 235 / 236}$ (Figure 6 and Supplemental Figure S1B). Other Akt substrates, including $\mathrm{p}-\mathrm{FoxO}^{\mathrm{S} 256}$ and $\mathrm{p}$ Prsa40 ${ }^{\mathrm{T} 246}$, did not change significantly. Consistent with the decreased ductal reaction in Rictor ${ }^{L K O}$ mice, the expression of biliary cell markers, such as Sox9, was decreased (Figure 6 and Supplemental Figure S1B).

Finally, to further investigate how the loss of mTORC2 influences liver injury and regeneration, mice were fed the DDC diet for 3 weeks and switched to regular mouse chow for additional 3 weeks to allow the mice to recover from DDC-induced liver injury. A significant decrease of CK19 $(+)$ cells after 3 weeks of recovery in both Rictor $^{\text {fl/f }}$ and Rictor $^{L K O}$ mice was observed (Supplemental Figure S6).

In summary, DDC treatment triggers cholestatic liver injury in both Rictor ${ }^{A l f l}$ and Rictor ${ }^{L K O}$ mice. However, loss of mTORC2 leads to reduced DDC-driven Akt activation and decreases oval cell expansion in vivo.

\section{mTORC2 from Progenitor or Biliary Epithelial Cells Drives Oval Cell Expansion in Vivo}

Albumin-Cre mice are responsible for the deletion of the floxed gene in liver hepatoblasts, leading to gene knockout in both hepatocytes and biliary epithelial cells in adult mice. $^{23}$ To further investigate the cellular population affected by mTORC2 deficiency during ductular reaction, we specifically deleted Rictor in adult hepatocytes. In brief, Rictor $^{\text {fl/fl}}$ mice were infected with AAV-TBG-Cre. Rictor ${ }^{\text {fl/fl}}$ littermates infected with AAV-TBG-Null vector were used as controls (Figure 7A). Three weeks after AAV injection, 

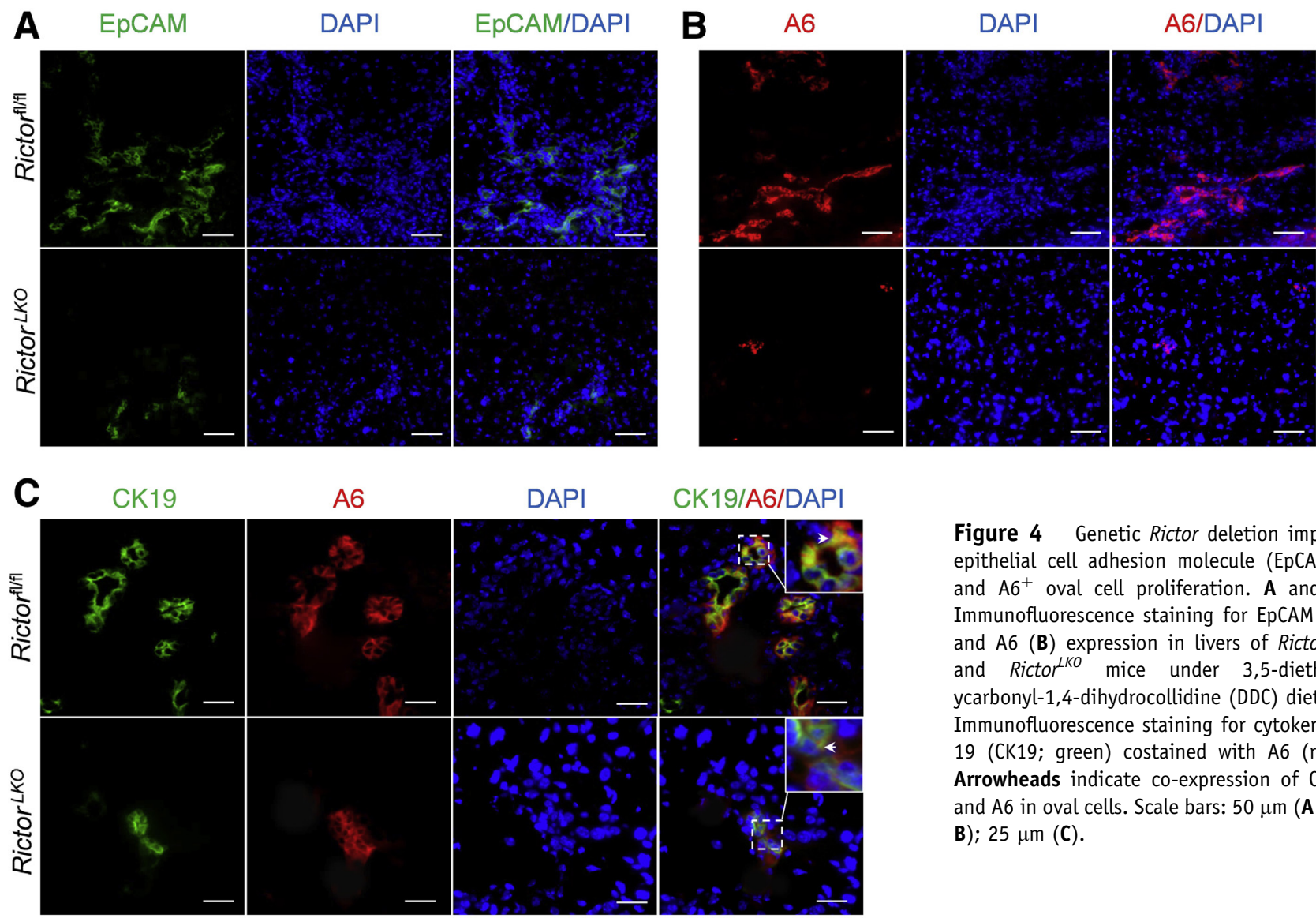

Figure 4 Genetic Rictor deletion impairs epithelial cell adhesion molecule (EpCAM) ${ }^{+}$ and $\mathrm{A}^{+}$oval cell proliferation. $\mathbf{A}$ and $\mathbf{B}$ : Immunofluorescence staining for $\operatorname{EpCAM}(\mathbf{A})$ and A6 (B) expression in livers of Rictor ${ }^{f l f l}$ and Rictor ${ }^{\perp K O}$ mice under 3,5-diethoxycarbonyl-1,4-dihydrocollidine (DDC) diet. C: Immunofluorescence staining for cytokeratin 19 (CK19; green) costained with A6 (red). Arrowheads indicate co-expression of CK19 and $A 6$ in oval cells. Scale bars: $50 \mu \mathrm{m}$ (A and B); $25 \mu \mathrm{m}$ (C).

mice were fed a DDC diet for additional 3 weeks. A significant similar degree of ductular reaction could be observed in DDC-treated AAV-TBG-Null and AAV-TBGCre injected Rictor $^{\text {flff }}$ mice (Figure 7B). Immunohistochemical staining of CK19 or A6 was performed to quantify the oval cell expansion in mice, and similar staining patterns for CK19 or A6 were obtained in the two mouse cohorts (Figure 7, B and C, and Supplemental Figure S7). Interestingly, Sox 9 staining revealed similar levels of Sox9 $(+)$ cells in periportal ductal-like cells. However, although numerous hepatocytes were found to be Sox9 (+) surrounding the periportal region, the number of Sox $9(+)$ hepatocytes was significantly lower in DDC-treated AAVTBG-Cre injected Rictor ${ }^{f / A}$ mice (Figure 7, D and E). The results suggest that hepatocyte mTORC2 controls Sox9 expression in hepatocytes, but not in oval cells. Western blot analysis revealed that AAV-TBG-Cre was effective in deleting Rictor in the adult liver, leading to decreased expression of $\mathrm{p}-\mathrm{Akt}^{\mathrm{S} 473}$, $\mathrm{p}-\mathrm{Rps}^{235 / 236}$, and $\mathrm{p}-\mathrm{mTOR}^{\mathrm{S} 2448}$ in these mice (Figure 7F and Supplemental Figure S1C).

Altogether, these findings suggest that liver progenitor cells or biliary epithelial cells, rather than hepatocytes, are the main cells types required for mTORC2 activation along ductular reaction in DDC-induced cholestatic liver injury.
The mTORC1/Fasn Signaling Is Dispensable in DDCInduced Ductular Reaction

Because mTORC1 is the major signaling event downstream of the mTORC2/Akt cascade and p-mTOR ${ }^{\mathrm{S} 2448}$ levels were significantly decreased in DDC-treated Rictor ${ }^{L K O}$ mice, we hypothesized that mTORC1 inhibition may also lead to decreased ductular reaction. To test this hypothesis, wildtype mice were subjected to daily rapamycin injection and DDC treatment (Supplemental Figure S8A). Additional mice were treated with vehicle and DDC as control. After 2 weeks of DDC treatment, similar oval cell expansion in vehicle and rapamycin-treated mice occurred (Supplemental Figure S8B). This observation was confirmed by CK19 immunohistochemistry (Supplemental Figure S8C). At the molecular level, as expected, rapamycin treatment effectively inhibited the levels of p-Rps6, the downstream effector of mTORC1 (Supplemental Figure S8D).

FASN-regulated de novo lipid biosynthesis is a major metabolic cascade downstream of mTORC1/Rps6. ${ }^{24}$ To test whether the lipogenic pathway is also dispensable for DDC-induced ductular reaction, liver-specific Fasn knockout $\left(\mathrm{Fash}^{L K O}\right)$ mice were generated by crossing $\mathrm{Al}$ bumin-Cre mice with Fasn ${ }^{f l f l}$ mice, as described in our previous publication (Supplemental Figure S9A). ${ }^{25}$ Thus, 

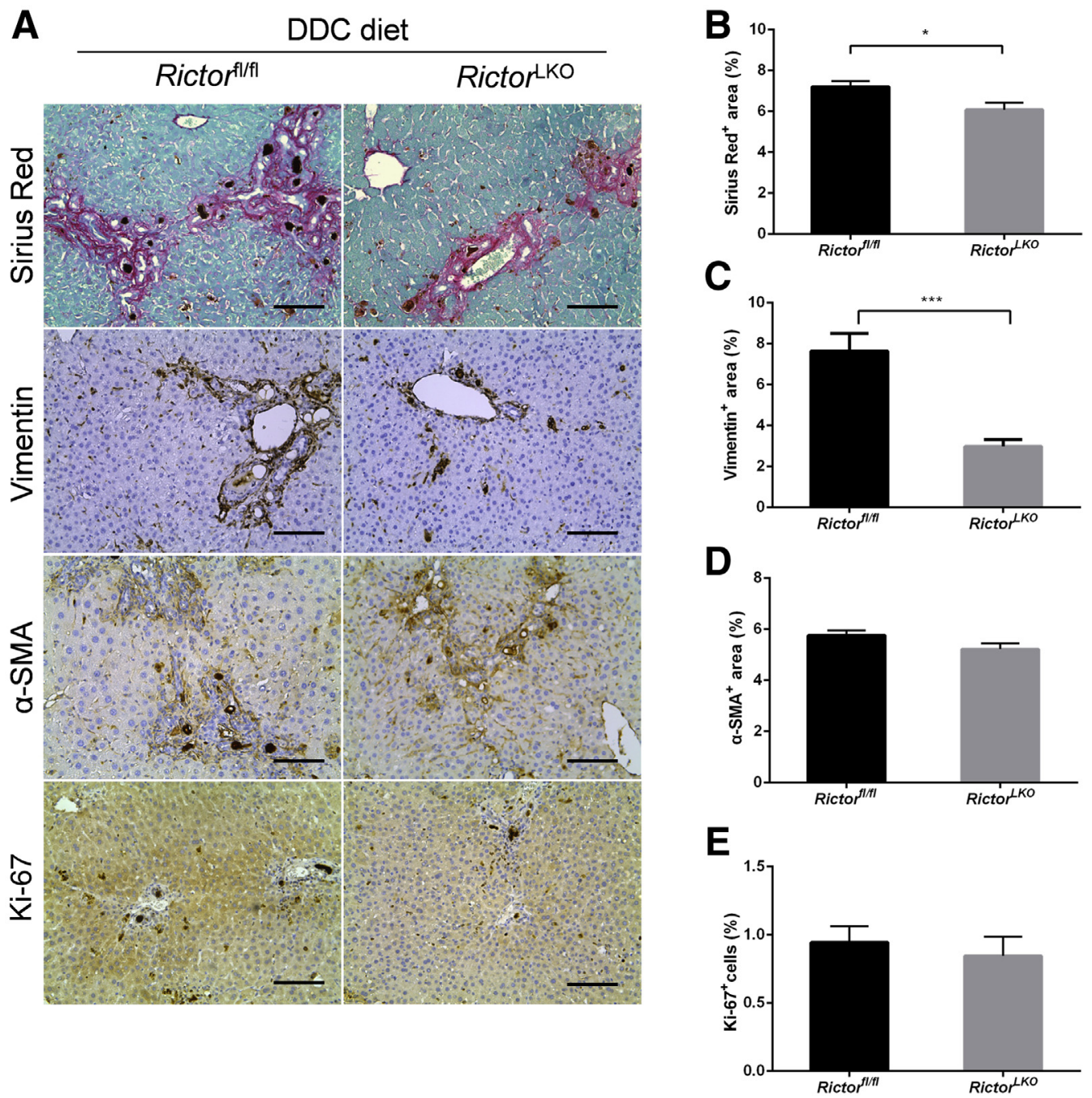

Figure 5 Ablation of Rictor may affect fibrosis but not hepatocyte proliferation. A: Picro Sirius Red staining and immunohistochemistry of vimentin, $\alpha-$ smooth muscle actin ( $\alpha$-SMA), and Ki-67 in the livers of Rictor ${ }^{f l f l}$ and Rictor ${ }^{1 K O}$ mice under 3,5-diethoxycarbonyl-1,4-dihydrocollidine (DDC) diet. B-E: Quantification of Picro Sirius Red (B), vimentin (C), $\alpha$-SMA (D), and Ki-67 (E) staining. At least three mice per group were assayed. Data are expressed as means \pm SEM (B-E). ${ }^{*} P<0.05,{ }^{* *} P<0.001$. Scale bars $=100 \mu \mathrm{m}(\mathbf{A})$.

Fasn $^{L K O}$ and control Fasn ${ }^{\text {flfl }}$ mice were fed DDC for 3 weeks. Fasn ${ }^{L K O}$ and $\mathrm{Fasn}^{f / / f}$ mice displayed a similar oval cell expansion pattern, as assessed by histopathologic analysis and CK19 immunohistochemistry (Supplemental Figure S9, B and C). Western blot analysis confirmed the effective deletion of Fasn in the mouse liver (Supplemental Figure S9D).

In summary, our study indicates that the mTORC2/Akt pathway regulates DDC-induced ductular reaction independent of the mTORC1/Rps6/Fasn cascade.

\section{Aberrant Notch but Not Yap Activation in DDC-Treated Rictor $^{\perp K O}$ Mice}

Finally, we investigated the possible pathways downstream of mTORC2 in regulating oval cell expansion in response to DDC treatment. As altered bile acid synthesis, gluconeogenesis, and lipogenesis have been shown to be the major metabolic pathways downstream of mTORC2 in the liver, the expression of genes involved in these pathways in DDC${\text { treated } \text { Rictor }^{\text {flfl }} \text { and Rictor }}^{L K O}$ mouse liver tissues by quantitative real-time RT-PCR were analyzed. All these genes exhibited similar expression levels in the two cohorts of mice (Supplemental Figure S4, B-D), suggesting that these metabolic processes are not the major events downstream of mTORC2 during DDC-induced liver injury. A recent investigation indicates that the Hippo/Yap cascade is involved in DDC-induced oval cell expansion. ${ }^{26}$ Additional studies suggest that Yap is a downstream effector of mTORC2. ${ }^{27,28}$ Thus, we investigated whether DDC-treated control and Rictor ${ }^{L K O}$ mouse liver tissues were characterized by activation of Yap by immunohistochemistry. Enhanced cytoplasmic and nuclear Yap expression was found in oval cells from DDC-treated control Rictor ${ }^{\text {fl/fl }}$ mouse livers. A similar Yap expression pattern characterized DDC-treated Rictor ${ }^{L K O}$ mouse liver tissues (Supplemental Figure S10A). Furthermore, no difference in the total levels of either Yap or its paralog Taz was detected 

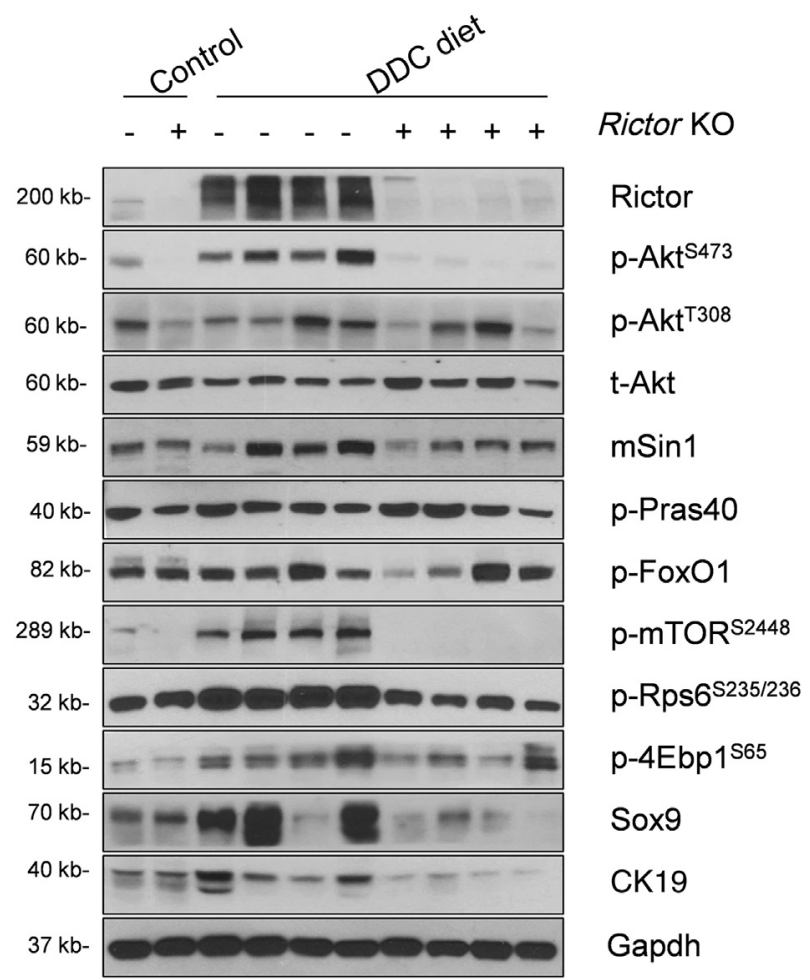

Figure 6 Ablation of Rictor reduces Akt signaling activation. Western blot analysis of the mTORC2/Akt signaling cascade in livers of Rictor ${ }^{f l / f l}$ and Rictor ${ }^{1 K O}$ mice under 3,5-diethoxycarbonyl-1,4-dihydrocollidine (DDC) diet. Glyceraldehyde-3-phosphate dehydrogenase (Gapdh) was used as loading control. 4Ebp1, eukaryotic translation initiation factor 4E-binding protein 1; CK19, cytokeratin 19; Fox01, forkhead box 01; K0, knockout; mSin1, mitogen-activated protein kinase associated protein 1; mTOR, mammalian target of rapamycin; p-, phosphorylated; Pras40, proline-rich AKT substrate of $40 \mathrm{kDa}$; Rictor, rapamycin-insensitive companion of mTOR; Rps6, ribosomal protein S6; Sox9, SRY (sex determining region $\mathrm{Y}$ )-box 9; $\mathrm{t}$-, total.

in the two mouse cohorts by Western blot analysis (Supplemental Figure S10B).

Notch signaling cascade is another pathway that has been implicated in oval cell expansion. ${ }^{29}$ We examined Notch pathway status in DDC-treated Rictor ${ }^{\text {fl/f }}$ and Rictor ${ }^{\text {LKO }}$ mouse liver tissues. Intriguingly, a significant decrease in Jag1 protein expression was observed in DDC-treated Rictor $^{L K O}$ mouse liver tissues (Supplemental Figure S10B). Consistently, quantitative real-time RT-PCR analysis confirmed the decreased mRNA expression of canonical Notch targets, such as Hes 1, Hes5, and HeyL1, in DDCtreated Rictor ${ }^{L K O}$ mouse livers (Supplemental Figure S10C).

Altogether, these results indicate loss of mTORC2 does not affect Hippo/Yap cascade, whereas it inhibits the Notch pathway, along DDC-induced ductular reaction.

\section{Discussion}

Recent studies have established mTOR complexes as essential modulator of numerous cellular processes, including cell metabolism and growth. mTOR functions in two distinct complexes: mTORC1, which is sensitive to rapamycin treatment; and mTORC2, which is insensitive to rapamycin. Although the role of mTORC1 in liver homeostasis, regeneration, and tumor development has been extensively investigated, the studies about mTORC2 in liver biology remain limited. As Rictor is a unique subunit of mTORC2, conditional Rictor knockout mice have been used to characterize the function of $\mathrm{mTORC} 2$ in various cell types. In the liver, studies on mTORC2 mainly focus on its role in hepatic metabolism and carcinogenesis. For instance, it was found that hepatic mTORC2 deletion leads to loss of $\mathrm{p}-\mathrm{Akt}^{\mathrm{S}}{ }^{773}$. This resulted in reduced activity of glucokinase and sterol regulatory element binding transcription factor 1 (Srebp1) proteins, leading to impaired glycolysis and lipogenesis in Rictor ${ }^{L K O}$ mice. $^{21}$ In agreement with the latter findings, Rictor ${ }^{L K O}$ mice failed to develop a fatty liver phenotype when fed a high-fat diet. ${ }^{30}$ In addition, Rictor ${ }^{\text {LKO }}$ mice exhibited alterations in insulin-induced Akt activation and insulin-driven mTORC1 activation, leading to glucose intolerance and insulin resistance. ${ }^{21}$ In human hepatocellular carcinoma, chromosomal gains of RICTOR were found in approximately $25 \%$ of patient samples, ${ }^{31}$ and high expression of RICTOR was associated with poor prognosis. ${ }^{32}$ In addition, inhibition of mTORC2 induced cell cycle arrest in hepatocellular carcinoma cell lines. ${ }^{33}$ Furthermore, loss of Rictor prevented sgPten/c-Met ${ }^{34}$ and c-Myc/MCL1 ${ }^{32}$ induced hepatocellular carcinoma formation in mice. Taken together, these findings revealed the key role of mTORC2 in the regulation of liver metabolism and carcinogenesis. However, the functional contribution of mTORC2 to liver regeneration has not been reported to date. In this article, we investigated how mTORC2 regulates liver regeneration after DDC-induced cholestatic liver injury using Rictor ${ }^{L K O}$ mice. We discovered that mTORC2/Akt signaling cascade is activated after DDC treatment in wildtype mice. More important, we found that loss of mTORC2 did not prevent DDC-induced cholestatic liver injury, as similar serologic features, including increased levels of alanine aminotransferase, aspartate aminotransferase, total bilirubin, direct bilirubin, taurocholic acid, deoxycholic acid, and total bile acid, were observed in both control and Rictor $^{L K O}$ mice. Although we were unable to measure bile flow rates in the DDC-treated mouse cohort, in a previous study, it was shown that DDC treatment does not alter bile flow rate in mice. ${ }^{35}$ It is well established that oval cell expansion is a major phenotype in the DDC-induced cholestatic liver injury model. We found that liver-specific ablation of Rictor significantly inhibited oval cell expansion in DDC fed mice, supporting a major function of the mTORC2 signaling cascade in this process. The present studies focus on DDC-induced cholestasis, but additional models, ${ }^{36}$ such as bile duct ligation model and $M d r 2^{-1-}$ mouse model, in combination with Rictor $^{L K O}$ mice, should be applied. These studies will provide additional support for the role of mTORC2 in cholestatic disease pathogenesis.

mTORC2 phosphorylates several AGC kinases, such as AKT proteins (AKT1, AKT2, and AKT3), SGKs (SGK1, 
A

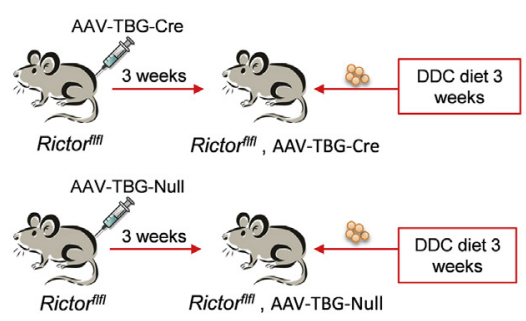

D

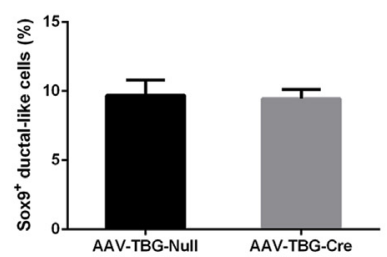

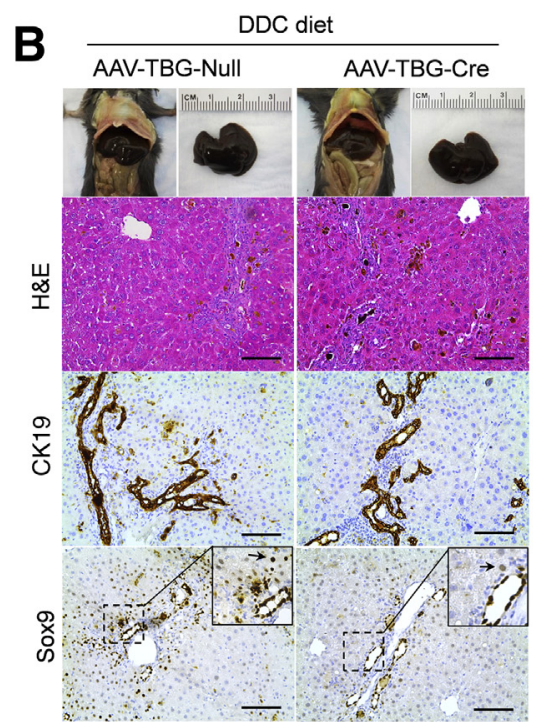

C

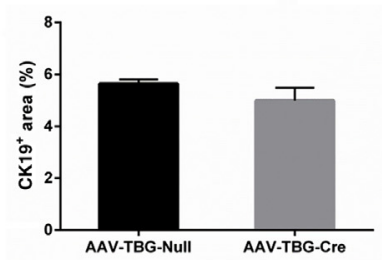

$\mathbf{E}$

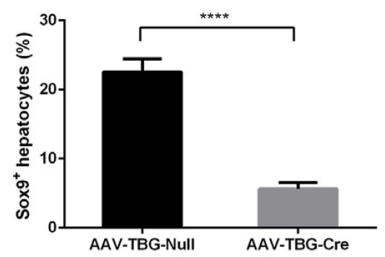

$\mathbf{F}$

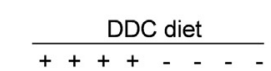

AAV-TBG-Null AAV-TBG-Cre

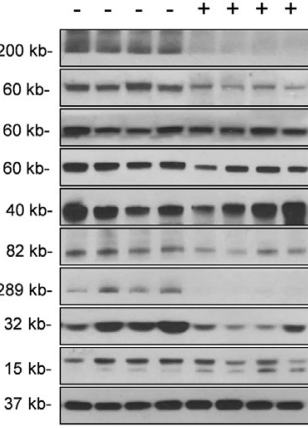

Rictor p-Akt ${ }^{S 43}$ p-Akt ${ }^{\top 308}$ t-Akt p-Pras40 p-FoxO1 p-mTOR ${ }^{\mathrm{S} 248}$ p-Rps6 $2235 / 236$ p-4Ebp 1\$65 Gapdh

Figure 7 Rictor deficiency in hepatocytes has no effect on ductular reaction. A: Scheme of the experiment. B: Gross image and hematoxylin and eosin (H\&E) and immunohistochemical staining of cytokeratin 19 (CK19) and sex-determining region Y-box 9 (Sox9) in the livers of AAV-Cre-Rictor and AAV-Null

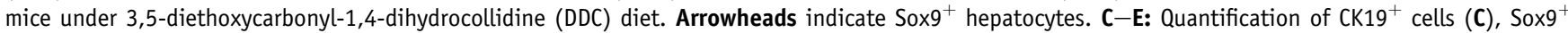
ductular-like cells (D), and Sox9 ${ }^{+}$hepatocytes (E). F: Western blot analysis of mTORC2/Akt signaling cascade in AAV-Cre-Rictor and AAV-Null mouse liver under DDC diet. Glyceraldehyde-3-phosphate dehydrogenase (Gapdh) was used as loading control. At least three mice per group were assayed. Data are expressed as means \pm SEM $(\mathbf{C}-\mathbf{E}) .{ }^{* * *} P<0.0001$. Scale bars $=100 \mu \mathrm{m}(\mathbf{B}) .4$ Ebp1, eukaryotic translation initiation factor 4E-binding protein; Fox01, forkhead box 01; mTOR, mammalian target of rapamycin; p-, phosphorylated; Pras40, proline-rich AKT substrate of $40 \mathrm{kDa}$; Rictor, rapamycin-insensitive companion of mTOR; Rps6, ribosomal protein S6; t-, total.

SGK2, and SGK3), and protein kinase C, leading to the activation of these kinases. Among these mTORC2 substrates, AKT kinases are considered the major downstream effectors of mTORC2. In the mouse liver, only Akt1 and Akt2, but not Akt3, are expressed. Between Akt1 and Akt2, it has been estimated that Akt2 represents approximately $85 \%$ of total Akt in the liver. Previous studies have shown that different Akt isoforms may have distinct roles in biological processes. For instance, Akt2 has been identified as the main Akt isoform downstream of insulin signaling in the liver, ${ }^{37,38}$ and it is required for loss of phosphatase and tensin homolog (Pten)-induced hepatic steatosis. ${ }^{39}$ Overexpression of constitutively activated Akt 2 in Rictor $^{L K O}$ mice rescued the metabolic defects observed in Rictor ${ }^{L K O}$ mice. ${ }^{21}$ Akt2 is also required for activated mutant form of PIK3CA or loss of Pten driven hepatocellular carcinoma formation in mice. ${ }^{40,41}$ The role of Akt1 in the liver has been only partially characterized. ${ }^{42}$ Recently, it was found that Akt1, but not Akt2, is the dominant Akt isoform in c-MYC driven liver carcinogenesis. ${ }^{32}$ On the other hand, it has been suggested that Akt1 and Akt2 have redundant roles in liver homeostasis, and concomitant deletion of Atkl and Akt2 is required to disrupt such equilibrium. ${ }^{43}$ Our current study suggests that Akt1 may be the principal Akt isoform required for oval cell expansion. This hypothesis could be tested via generation of liver-specific Aktl, Akt2, and Akt1/ $A k t 2$ double-knockout mice and subjecting them to DDC feeding.

To further investigate the molecular mechanisms whereby the mTORC2/Akt pathway modulates DDC-induced cholestatic liver injury, we analyzed the expression of downstream signaling molecules in DDC-treated control and Rictor $^{L K O}$ mice. We discovered a significant decreased expression of p-mTOR, p-Rps6, and p-4Ebp1 proteins, 
suggesting a decrease in the mTORC1 activity. In contrast, other known mTORC2/Akt substrates, including $p$ Pras $40^{\mathrm{T} 246}$ and $\mathrm{p}-\mathrm{FoxO} 1^{\mathrm{S} 256}$, were not affected by the loss of Rictor. These data suggest that mTORC1 may be the key signaling complex downstream of mTORC2/Akt1 in cholestatic liver injury. Interestingly, using rapamycin treatment and liver-specific Fasn knockout mice, we demonstrate that Rps6/Fasn signaling cascade is dispensable for DDC-induced oval cell expansion. Rapamycin treatment inhibits p-Rps6 downstream of mTORC1, but not p4Ebp1. ${ }^{44}$ Thus, it is possible that 4Ebp1/eukaryotic translation initiation factor $4 \mathrm{E}$-mediated translation is the major signaling event downstream of mTORC2/Akt during cholestatic liver injury. Recent studies have demonstrated the key roles of Hippo and Notch cascades in oval cell expansion. ${ }^{26-29}$ Our investigation shows that the Notch cascade, but not the Hippo signaling, is down-regulated in DDC-treated Rictor ${ }^{L K O}$ mouse liver tissues. More important, Jag1, the predominant Notch ligand in the liver, was expressed at lower levels in DDC-treated Rictor ${ }^{L K O}$ livers. Further analysis suggested that Jag1 mRNA levels were equivalent in DDC-treated control and Rictor $^{L K O}$ liver tissues (Supplemental Figure S10C), indicating that Jag1 is regulated at the post-transcriptional level by mTORC2. Overall, it is tempting to hypothesize that mTORC2/Akt regulates 4Ebp1/eukaryotic translation initiation factor 4E downstream of mTORC1, leading to the increased protein synthesis of Jag1, elevated Notch signaling, and, eventually, oval cell expansion after DDC treatment. Clearly, additional studies are required to investigate this hypothesis. The results from these studies will provide novel mechanistic insights into how mTORC2/Akt and Notch signaling cascades interact during cholestatic liver injury-induced oval cell expansion.

\section{Acknowledgments}

We thank Dr. Clay F. Semenkovich (Washington University, St. Louis, MO) for providing Fasn ${ }^{f l f}$ mice; and Dr. Valentina M. Factor (Laboratory of Experimental Carcinogenesis, National Cancer Institute, NIH) for the A6 antibody used in the study.

\section{Supplemental Data}

Supplemental material for this article can be found at http://doi.org/10.1016/j.ajpath.2020.03.010.

\section{References}

1. Hirschfield GM, Heathcote EJ, Gershwin ME: Pathogenesis of cholestatic liver disease and therapeutic approaches. Gastroenterology 2010, 139:1481-1496

2. European Association for the Study of the Liver: EASL Clinical Practice Guidelines: management of cholestatic liver diseases. J Hepatol 2009, 51:237-267
3. Mariotti V, Strazzabosco M, Fabris L, Calvisi DF: Animal models of biliary injury and altered bile acid metabolism. Biochim Biophys Acta Mol Basis Dis 2018, 1864:1254-1261

4. Fickert P, Thueringer A, Moustafa T, Silbert D, Gumhold J, Tsybrovskyy O, Lebofsky M, Jaeschke H, Denk H, Trauner M: The role of osteopontin and tumor necrosis factor alpha receptor-1 in xenobiotic-induced cholangitis and biliary fibrosis in mice, laboratory investigation. J Tech Methods Pathol 2010, 90:844-852

5. Fickert P, Stoger U, Fuchsbichler A, Moustafa T, Marschall HU, Weiglein AH, Tsybrovskyy O, Jaeschke H, Zatloukal K, Denk H, Trauner M: A new xenobiotic-induced mouse model of sclerosing cholangitis and biliary fibrosis. Am J Pathol 2007, 171:525-536

6. Jakubowski A, Ambrose C, Parr M, Lincecum JM, Wang MZ, Zheng TS, Browning B, Michaelson JS, Baetscher M, Wang B, Bissell DM, Burkly LC: TWEAK induces liver progenitor cell proliferation. J Clin Invest 2005, 115:2330-2340

7. Li Z, Zhang J, Mulholland M, Zhang W: mTOR activation protects liver from ischemia/reperfusion-induced injury through NF-kappaB pathway. FASEB J 2017, 31:3018-3026

8. Panasyuk G, Patitucci C, Espeillac C, Pende M: The role of the mTOR pathway during liver regeneration and tumorigenesis. Ann Endocrinol 2013, 74:121-122

9. Saxton RA, Sabatini DM: mTOR signaling in growth, metabolism, and disease. Cell 2017, 168:960-976

10. Li J, Xu Z, Jiang L, Mao J, Zeng Z, Fang L, He W, Yuan W, Yang J, Dai C: Rictor/mTORC2 protects against cisplatin-induced tubular cell death and acute kidney injury. Kidney Int 2014, 86:86-102

11. Hallowell RW, Collins SL, Craig JM, Zhang Y, Oh M, Illei PB, Chan-Li Y, Vigeland CL, Mitzner W, Scott AL, Powell JD, Horton MR: mTORC2 signalling regulates M2 macrophage differentiation in response to helminth infection and adaptive thermogenesis. Nat Commun 2017, 8:14208

12. Laplante M, Sabatini DM: mTOR signaling in growth control and disease. Cell 2012, 149:274-293

13. Sarbassov DD, Ali SM, Sabatini DM: Growing roles for the mTOR pathway. Curr Opin Cell Biol 2005, 17:596-603

14. Onori P, Alvaro D, Floreani AR, Mancino MG, Franchitto A, Guido M, Carpino G, De Santis A, Angelico M, Attili AF, Gaudio E: Activation of the IGF1 system characterizes cholangiocyte survival during progression of primary biliary cirrhosis. J Histochem Cytochem 2007, 55:327-334

15. Postic C, Shiota M, Niswender KD, Jetton TL, Chen Y, Moates JM, Shelton KD, Lindner J, Cherrington AD, Magnuson MA: Dual roles for glucokinase in glucose homeostasis as determined by liver and pancreatic beta cell-specific gene knock-outs using Cre recombinase. J Biol Chem 1999, 274:305-315

16. Tang F, Wu Q, Ikenoue T, Guan KL, Liu Y, Zheng P: A critical role for Rictor in T lymphopoiesis. J Immunol 2012, 189:1850-1857

17. Chakravarthy MV, Zhu Y, Lopez M, Yin L, Wozniak DF, Coleman $\mathrm{T}$, Hu Z, Wolfgang $\mathrm{M}$, Vidal-Puig A, Lane MD, Semenkovich CF: Brain fatty acid synthase activates PPARalpha to maintain energy homeostasis. J Clin Invest 2007, 117:2539-2552

18. Fan L, Xu C, Wang C, Tao J, Ho C, Jiang L, Gui B, Huang S, Evert M, Calvisi DF, Chen X: Bmil is required for hepatic progenitor cell expansion and liver tumor development. PLoS One 2012, 7: e46472

19. Nantasanti S, Toussaint MJ, Youssef SA, Tooten PC, de Bruin A: Rb and p53 liver functions are essential for xenobiotic metabolism and tumor suppression. PLoS One 2016, 11:e0150064

20. Tirnitz-Parker JE, Tonkin JN, Knight B, Olynyk JK, Yeoh GC: Isolation, culture and immortalisation of hepatic oval cells from adult mice fed a choline-deficient, ethionine-supplemented diet. Int J Biochem Cell Biol 2007, 39:2226-2239

21. Hagiwara A, Cornu M, Cybulski N, Polak P, Betz C, Trapani F, Terracciano L, Heim MH, Ruegg MA, Hall MN: Hepatic mTORC2 activates glycolysis and lipogenesis through Akt, glucokinase, and SREBP1c. Cell Metab 2012, 15:725-738 
22. Wang X, Foster M, Al-Dhalimy M, Lagasse E, Finegold M, Grompe M: The origin and liver repopulating capacity of murine oval cells. Proc Natl Acad Sci U S A 2003, 100 Suppl 1:11881-11888

23. Lemaigre FP: Determining the fate of hepatic cells by lineage tracing: facts and pitfalls. Hepatology 2015, 61:2100-2103

24. Bruning U, Morales-Rodriguez F, Kalucka J, Goveia J, Taverna F, Queiroz KCS, Dubois C, Cantelmo AR, Chen R, Loroch S, Timmerman E, Caixeta V, Bloch K, Conradi LC, Treps L, Staes A, Gevaert K, Tee A, Dewerchin M, Semenkovich CF, Impens F, Schilling B, Verdin E, Swinnen JV, Meier JL, Kulkarni RA, Sickmann A, Ghesquiere B, Schoonjans L, Li X, Mazzone M, Carmeliet P: Impairment of angiogenesis by fatty acid synthase inhibition involves mTOR malonylation. Cell Metab 2018, 28: 866-880.e815

25. Che L, Chi W, Qiao Y, Zhang J, Song X, Liu Y, Li L, Jia J, Pilo MG, Wang J, Cigliano A, Ma Z, Kuang W, Tang Z, Zhang Z, Shui G, Ribback S, Dombrowski F, Evert M, Pascale RM, Cossu C, Pes GM, Osborne TF, Calvisi DF, Chen X, Chen L: Cholesterol biosynthesis supports the growth of hepatocarcinoma lesions depleted of fatty acid synthase in mice and humans. Gut 2020, 69:177-186

26. Planas-Paz L, Sun T, Pikiolek M, Cochran NR, Bergling S, Orsini V, et al: YAP, but not RSPO-LGR4/5, signaling in biliary epithelial cells promotes a ductular reaction in response to liver injury. Cell Stem Cell 2019, 25:39-53.e10

27. Artinian N, Cloninger C, Holmes B, Benavides-Serrato A, Bashir T, Gera J: Phosphorylation of the hippo pathway component AMOTL2 by the mTORC2 kinase promotes YAP signaling, resulting in enhanced glioblastoma growth and invasiveness. J Biol Chem 2015, 290:19387-19401

28. Gui Y, Li J, Lu Q, Feng Y, Wang M, He W, Yang J, Dai C: Yap/Taz mediates mTORC2-stimulated fibroblast activation and kidney fibrosis. J Biol Chem 2018, 293:16364-16375

29. Spee B, Carpino G, Schotanus BA, Katoonizadeh A, Vander Borght S, Gaudio E, Roskams T: Characterisation of the liver progenitor cell niche in liver diseases: potential involvement of Wnt and Notch signalling. Gut 2010, 59:247-257

30. Yuan M, Pino E, Wu L, Kacergis M, Soukas AA: Identification of Akt-independent regulation of hepatic lipogenesis by mammalian target of rapamycin (mTOR) complex 2. J Biol Chem 2012, 287: 29579-29588

31. Villanueva A, Chiang DY, Newell P, Peix J, Thung S, Alsinet C, Tovar V, Roayaie S, Minguez B, Sole M, Battiston C, Van Laarhoven S, Fiel MI, Di Feo A, Hoshida Y, Yea S, Toffanin S, Ramos A, Martignetti JA, Mazzaferro V, Bruix J, Waxman S, Schwartz M, Meyerson M, Friedman SL, Llovet JM: Pivotal role of mTOR signaling in hepatocellular carcinoma. Gastroenterology 2008, 135:1972-1983.e11

32. Xu Z, Xu M, Liu P, Zhang S, Shang R, Qiao Y, Che L, Ribback S, Cigliano A, Evert K, Pascale RM, Dombrowski F, Evert M, Chen X, Calvisi DF: The mTORC2-Akt1 cascade is crucial for c-Myc to promote hepatocarcinogenesis in mice and humans. Hepatology 2019, 70:1600-1613

33. Chen BW, Chen W, Liang H, Liu H, Liang C, Zhi X, Hu LQ, Yu XZ, Wei T, Ma T, Xue F, Zheng L, Zhao B, Feng XH, Bai XL, Liang TB: Inhibition of mTORC2 induces cell-cycle arrest and enhances the cytotoxicity of doxorubicin by suppressing MDR1 expression in HCC cells. Mol Cancer Ther 2015, 14:1805-1815

34. Xu Z, Hu J, Cao H, Pilo MG, Cigliano A, Shao Z, Xu M, Ribback S, Dombrowski F, Calvisi DF, Chen X: Loss of Pten synergizes with cMet to promote hepatocellular carcinoma development via mTORC2 pathway. Exp Mol Med 2018, 50:e417

35. Soltis EE, Newman PS: Age-dependent salt-induced hypertension in the rat: prevention with DSP-4, a selective noradrenergic neurotoxin. Clin Exp Hypertens A 1991, 13:557-572

36. Pollheimer MJ, Fickert P: Animal models in primary biliary cirrhosis and primary sclerosing cholangitis. Clin Rev Allergy Immunol 2015, 48:207-217

37. Cho H, Mu J, Kim JK, Thorvaldsen JL, Chu Q, Crenshaw EB 3rd, Kaestner KH, Bartolomei MS, Shulman GI, Birnbaum MJ: Insulin resistance and a diabetes mellitus-like syndrome in mice lacking the protein kinase Akt2 (PKB beta). Science 2001, 292: $1728-1731$

38. Leavens KF, Easton RM, Shulman GI, Previs SF, Birnbaum MJ: Akt2 is required for hepatic lipid accumulation in models of insulin resistance. Cell Metab 2009, 10:405-418

39. He L, Hou X, Kanel G, Zeng N, Galicia V, Wang Y, Yang J, Wu H, Birnbaum MJ, Stiles BL: The critical role of AKT2 in hepatic steatosis induced by PTEN loss. Am J Pathol 2010, 176: 2302-2308

40. Wang C, Che L, Hu J, Zhang S, Jiang L, Latte G, Demartis MI, Tao J, Gui B, Pilo MG, Ribback S, Dombrowski F, Evert M, Calvisi DF, Chen X: Activated mutant forms of PIK3CA cooperate with RasV12 or c-Met to induce liver tumour formation in mice via AKT $2 / \mathrm{m}$ TORC1 cascade. Liver Int 2016, 36:1176-1186

41. Galicia VA, He L, Dang H, Kanel G, Vendryes C, French BA, Zeng N, Bayan JA, Ding W, Wang KS, French S, Birnbaum MJ, Rountree CB, Stiles BL: Expansion of hepatic tumor progenitor cells in Pten-null mice requires liver injury and is reversed by loss of AKT2. Gastroenterology 2010, 139:2170-2182

42. Fan B, Yu Y, Zhang Y: PI3K-Akt1 expression and its significance in liver tissues with chronic fluorosis. Int J Clin Exp Pathol 2015, 8: $1226-1236$

43. Wang Q, Yu WN, Chen X, Peng XD, Jeon SM, Birnbaum MJ, Guzman G, Hay N: Spontaneous hepatocellular carcinoma after the combined deletion of Akt isoforms. Cancer Cell 2016, 29: $523-535$

44. Choo AY, Yoon SO, Kim SG, Roux PP, Blenis J: Rapamycin differentially inhibits S6Ks and 4E-BP1 to mediate cell-type-specific repression of mRNA translation. Proc Natl Acad Sci U S A 2008, 105:17414-17419 